Gut, 1988, 29, 795-801

\title{
Attachment of Giardia lamblia to rat intestinal epithelial cells
}

\author{
PHillipA M G INGE, C M EDSON, AND M J G FARTHING \\ From the Department of Gastroenterology, St Bartholomew's Hospital, London and Department of \\ Pathology, Tufts University School of Medicine, Boston MA, USA
}

SUMMARY The human enteric protozoan, Giardia lamblia, has surface membrane lectin activity which mediates parasite adherence to erythrocytes. To determine whether an intestinal binding site exists for this lectin we have studied the interaction in vitro between axenically cultured Giardia trophozoites and isolated rat intestinal epithelial cells. Scanning electron microscopy showed that Giardia attached to the apical microvillus membrane and basolateral membrane of rat enterocytes. Any location on the parasite surface could mediate attachment without predeliction for the ventral disc. Trophozoites attached more avidly to jejunal compared with colonic epithelial cells. Attachment was inhibited at $4^{\circ} \mathrm{C}$, by sugars and glycoproteins containing D-mannosyl residues and by subagglutinating concentrations of anti-Giardia rabbit serum and two monoclonal antibodies, all with reactivity to parasite surface membrane determinants. Trypsinisation of trophozoites also reduced attachment but the ability to attach was rapidly restored after returning trophozoites to TYI-S culture medium for $4 \mathrm{~h}$ at $37^{\circ} \mathrm{C}$. Attachment was unaltered by the presence of the microfilament inhibitor cytochalasin $\mathrm{B}$ and in the absence of $\mathrm{Ca}^{++}$and $\mathrm{Mg}^{++}$ions. These findings support previous work that Giardia possesses a surface membrane mannose binding lectin and indicate that appropriate binding sites are present on rat intestinal epithelial cells. This lectin may play a part in mediating adherence of Giardia to mammalian intestine and could be a target for host immune defence.

Adherence of microbial enteropathogens to host intestinal epithelium is considered to be a critical step in the pathogenesis of many intestinal infections. ${ }^{1}$ Giardia is commonly found in close proximity to the small intestinal mucosa and when in the ventral surface down position, it is generally considered that the parasite attaches to intestinal epithelial cells by either a suction force generated beneath the ventral disc by the propulsive efforts of the ventral flagella (hydrodynamic theory), ${ }^{2}$ by mechanical processes related to contractile protein elements of the ventral disc and ventrolateral flange,,$^{3-5}$ or by a combination of the two mechanisms.

Giardia may be found in other orientations with respect to the gut mucosa such as the dorsal surface down position ${ }^{6}$ as seen in one of our patients (Fig. 1).

Address for correspondence: M J G Farthing MD, MRCP. Department of Gastroenterology, St Bartholomew's Hospital. West Smithfield, London EC1A 7BE.

Received for publication 3 December 1987
This suggests that parasite surface membrane determinants may also be involved in the attachment process. We have shown previously using mammalian erythrocytes as a model target for attachment, that Giardia lamblia, like some bacteria and other protozoa, has lectin activity associated with its surface membrane with specificities for D-glucosyl and D-mannosyl residues.? To discover the possible relevance of this attachment mechanism in the pathogenesis of giardiasis, we have now investigated the interaction between Giardia and isolated mammalian intestinal epithelial cells to determine whether an intestinal receptor exists for this putative lectin in gut epithelium.

\section{Methods}

CULTURE AND HARVESTING OF GIARDIA TROPHOZOITES

Giardia lamblia trophozoites, Portland 1 strain were 


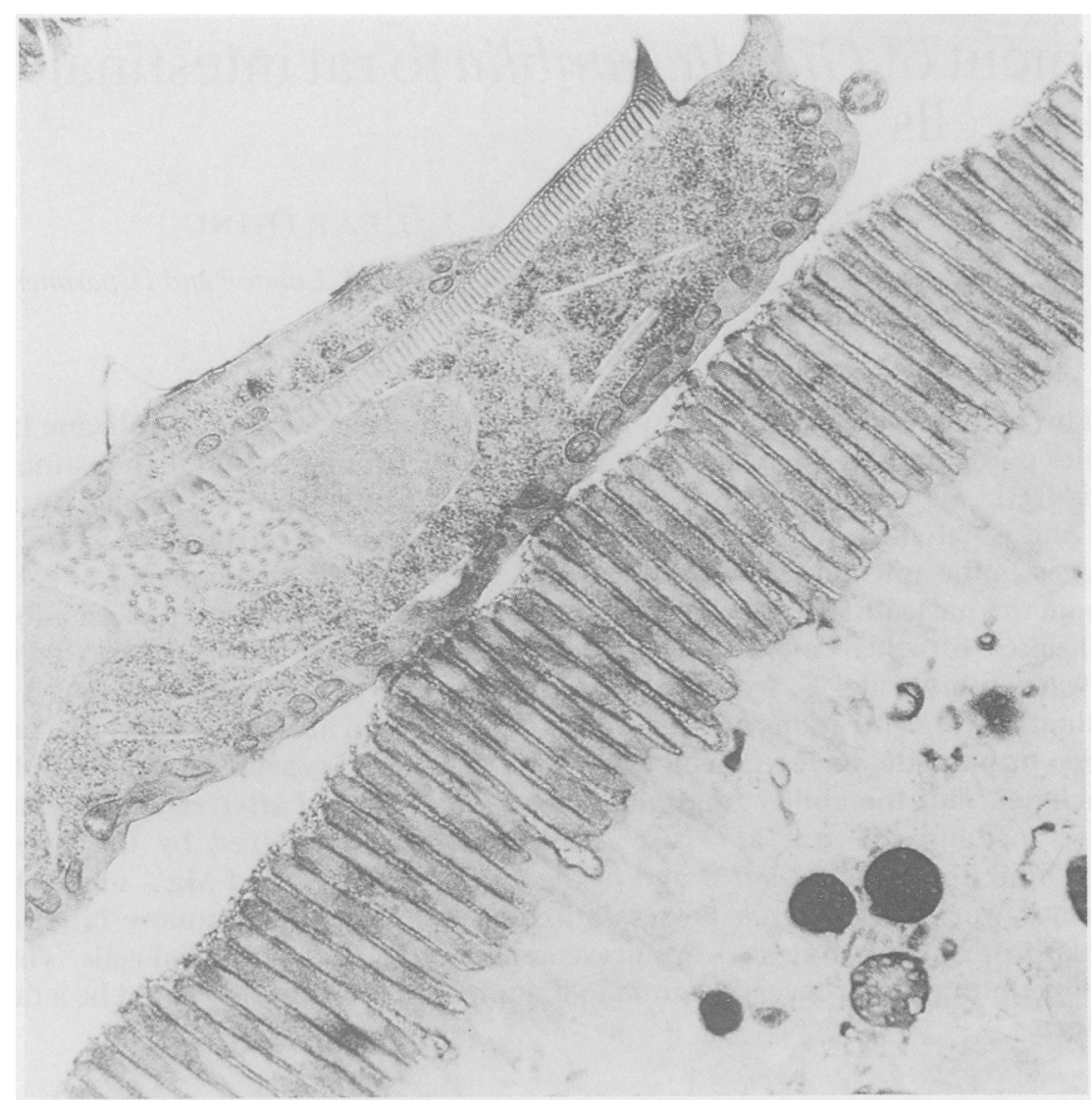

Fig. 1 Transmission electron micrograph of human jejunal mucosa from a patient with acute giardiasis. A Giardia lamblia trophozoite appears to be attached at several sites on its dorsal surface to the microvillus membrane of a jejunal enterocyte.

cultured in screwcap borosilicate glass tubes $(16 \times 125$ $\mathrm{mm}$ ) in modified TYI-S medium as described previously. ${ }^{7 x}$ New cultures were initiated with $1-5 \times 10^{5}$ trophozoites harvested during late log phase growth. Non-adherent parasites were discarded with the culture medium, the remainder were harvested with chilled $10 \mathrm{mM}$ hepes-saline buffer $(\mathrm{pH} 7 \cdot 2)$ containing $2 \mathrm{mmol} / \mathrm{l} \mathrm{CaCl}$ (HSCB). Tubes were then chilled on ice for 10 minutes after which liberated trophozoites were centrifuged $500 \mathrm{~g}$ for five minutes at $4^{\circ} \mathrm{C}$. Trophozoites were washed twice more and resuspended in HSCB to give a final concentration of approximately $1-5 \times 10^{\circ}$ trophozoites per $\mathrm{ml}$.

\section{ISOLATION OFRAT INTESTINAL EPITHELIAL}

CELIS

Rat small intestinal epithelial cells (enterocytes) were isolated by citrate/EDTA elution according to the method of Weiser" as used by us previously. ${ }^{\prime \prime}$ In the majority of experiments enterocytes were pooled and passed through a glass wool column to remove enterocyte clumps. In selected experiments, villus cell and crypt cell fractions were pooled separately as described previously. ${ }^{10}$ The resulting single cell preparation was resuspended in HSCB to give a final concentration of $1-5 \times 10^{6}$ enterocytes per ml. An identical approach was used to obtain epithelial cells from rat ileum and colon. For ileal cell preparations the distal $30 \mathrm{~cm}$ of small intestine was used and for colonic cells the entire colon was excised. Viability of epithelial cells assessed by trypan blue $(0.05 \%)$ exclusion was $75.5(10.4) \%$ (mean (SEM) for six experiments).

\section{GIARDIA INTESTINAL EPITHELIAL CELL}

\section{ATTACHMENT ASSAY}

We developed a visual attachment assay similar to previous assays used for quantification of attachment of bacteria and protozoa to target cells. ${ }^{11-13}$ Giardia trophozoites $\left(10^{5}\right)$ and isolated enterocytes $\left(10^{5}\right)$ were 
incubated in $100 \mu \mathrm{l}$ HSCB in 96 well microplates in air at $37^{\circ} \mathrm{C}$ for up to 60 minutes with continuous gentle shaking. At the end of the incubation the proportion of parasites attached to enterocytes was determined by phase contrast microscopy (Zeiss ID02, $\times 200$ magnification) by a 'blind' observer who was unaware of the experimental conditions. Ten random high power fields were systematically examined and the number of parasites attached to intestinal cells were counted and expressed as a percentage of the total number of trophozoites in that same field. To differentiate between parasites that were actually attached to an enterocyte and those in close proximity, the suspension was gently agitated before examination of each high power field. Preparations of Giardia trophozoites and enterocytes were fixed in $3 \%$ buffered glutaraldehyde and prepared for scanning electron microscopy. ${ }^{14}$

CHARACTERISATION OF GIARDIA INTESTINAL

EPITHELIAL CELL INTERACTION

The effect of time (0-60 min) and temperature (4 and $37^{\circ} \mathrm{C}$ ) on attachment of Giardia to gut epithelial cells were studied. Dependence on the presence of divalent cation $\left(\mathrm{Ca}^{++}, \mathrm{Mg}^{++}\right)$was investigated by adding $5 \mathrm{mM}$ EDTA or EGTA to incubations. Site specificity of attachment was investigated using epithelial cells from jejunum, ileum and colon. Carbohydrate inhibition studies were undertaken using the standard attachment assay procedure but with the addition of a wide range of simple and complex sugars $(<0 \cdot 1 \mathrm{M})$ and glycoproteins $(<10$ $\mathrm{mg} / \mathrm{ml})$. $^{?}$

Protease sensitivity of the putative parasite surface ligand was examined by exposing Giardia trophozoites to trypsin (Sigma Type III, Sigma Chemical Company, Poole, Dorset) at a concentration of $1 \mathrm{mg} / \mathrm{ml}$ at $37^{\circ} \mathrm{C}$ for five to 60 minutes. The reaction was stopped by addition of excess cold HSCB after which trophozoites were washed twice in HSCB. Viability of trophozoites after trypsin treatment was $99.5(0.8) \%$ (mean (SE), $\mathrm{n}=5$ ) by trypan blue exclusion and flagellar motility was maintained. Ability of trypsin treated trophozoites to attach to enterocytes was assessed in the standard attachment assay, (1) immediately after trypsin treatment and (2) after reculturing trypsin treated trophozoites in TYI$\mathrm{S}$ medium for four hours (less than one generation time). ${ }^{15}$

To determine whether cytoskeleton contractility, notably microfilament function was required for attachment of Giardia to enterocytes, trophozoites were exposed to the microfilament inhibitor cytochalasin B $(10 \mu \mathrm{g} / \mathrm{ml}$ in $0.4 \%$ dimethyl sulphoxide, 30 minutes at $37^{\circ} \mathrm{C}$ ) and then incubated with enterocytes for a further 30 minutes under the conditions of the standard attachment assay. In a previous study this treatment protocol markedly inhibited parasite attachment to glass and polystyrene surfaces although flagellar motility was still evident. ${ }^{16}$

To define further the possible role of a surface membrane determinant for parasite attachment to gut epithelium, the effect of anti-Giardia antibodies directed towards Giardia surface membrane were examined in the Giardia-enterocyte attachment assay. Anti-Giardia antiserum was raised in rabbits against live $G$ lamblia Portland 1 strain trophozoites. Murine monoclonal antibodies to $G$ lamblia were prepared as described previously. ${ }^{17}$ 18 One antibody, denoted GL-1 (IgGl) reacts with the entire parasite surface and immunoprecipitates an $80000-\mathrm{Mr}$ surface protein. ${ }^{1 \times}$ Indirect immunofluorescence studies show that a second monoclonal antibody GL-2 (IgG2a), is directed primarily towards a cytoplasmic determinant but with some surface membrane expression (unpublished observations). The anti-Giardia rabbit antiserum and the two monoclonal antibodies used in this study agglutinated live trophozoites confirming their activity towards surface membrane determinants. Their agglutination titres were determined by adding trophozoites $\left(1-5 \times 10^{5}\right)$ to doubling dilutions of heat inactivated $\left(56^{\circ} \mathrm{C}, 30 \mathrm{~min}\right)$ antibodies in 96 well flat bottom microplates. Subagglutination concentrations of antibodies were used in Giardia enterocyte inhibition studies. At these concentrations, parasite motility and growth in axenic culture were unimpaired (unpublished observations).

STATISTICAL ANALYSIS

Differences between observations were evaluated by a two-tailed $t$-test.

\section{Results}

SCANNING EIECTRONMICROSCOPY

Giardia trophozoites attached to microvillus and basolateral membranes of rat entereocytes (Fig. 2). Survey of random high power fields containing a total of 50 trophozoites showed that $18(36 \%)$ were attached to microvillus membrane and $32(64 \%)$ to basolateral membrane. All sites on the parasite surface were seen to be involved in the attachment process and in particular there was no predeliction for ventral structures notably the ventral disc.

\section{CHARACTERISATION OF GIARDIA-ENTEROCYTE ATTACHMENT}

Attachment of Giardia to enterocytes was time and temperature dependent (Fig. 3). The proportion of trophozoites attached to enterocytes increased during the first 30 minutes of the incubation at $37^{\circ} \mathrm{C}$ 


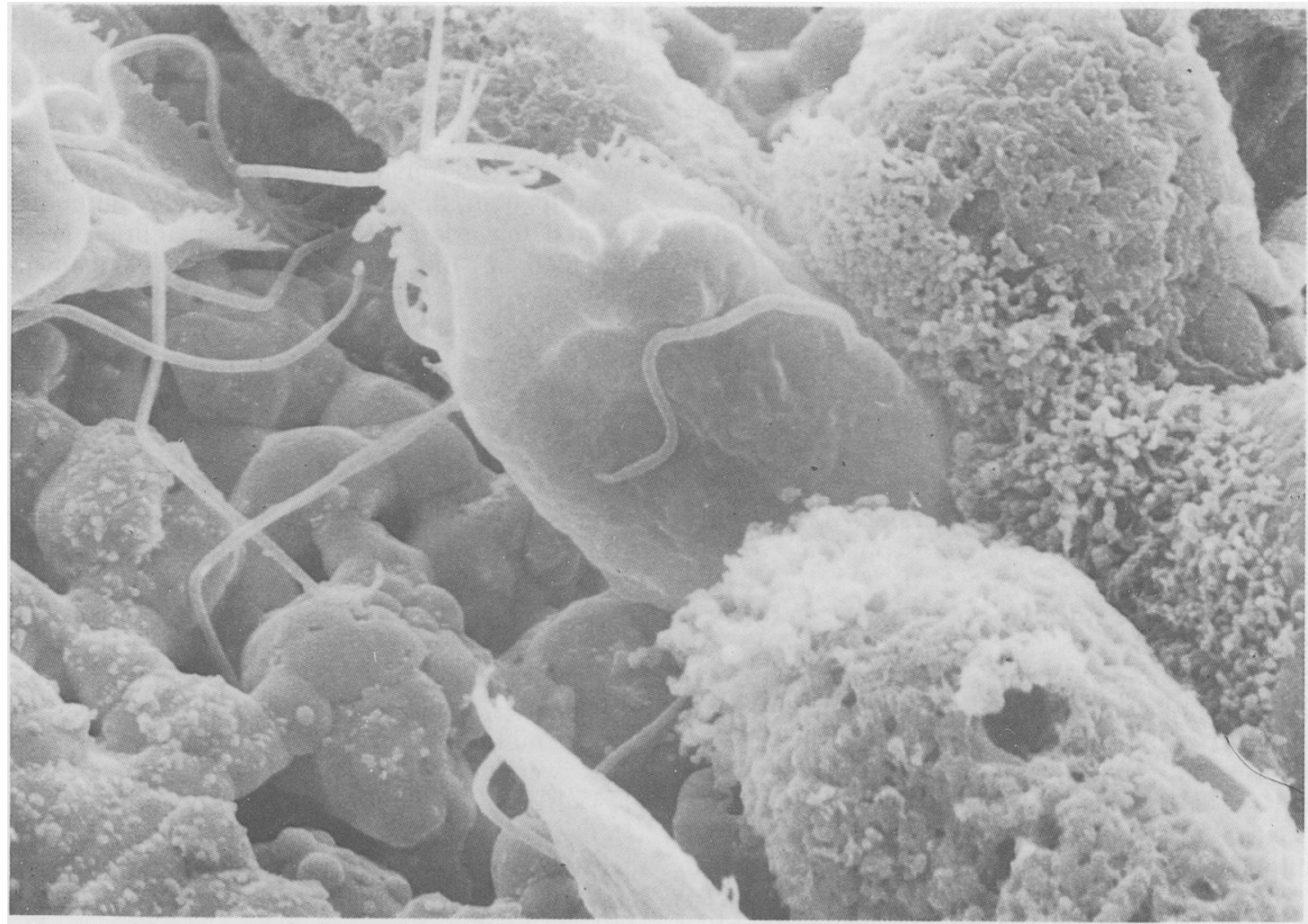

Fig. 2 Scanning electron micrograph of a Giardia trophozoite attached to the microvillus membrane of four isolated rat jejunal enterocytes.

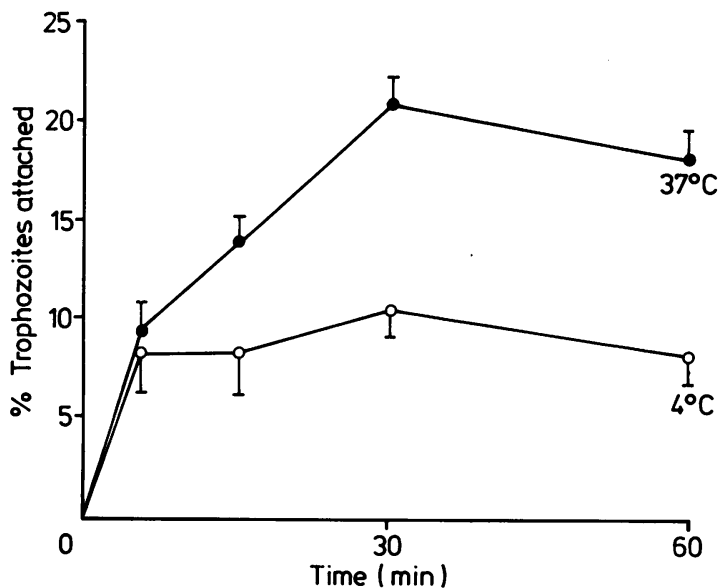

Fig. 3 Effect of time and temperature of incubation on attachment of axenically cultivated Giardia lamblia trophozoites to isolated rat jejunal enterocytes. Attachment is expressed as the proportion of the total number trophozoites present attached to epithelial cells. Each point represents the mean $(S E), n=5$. and then reached a plateau. Attachment was substantially reduced when the incubation was done at $4^{\circ} \mathrm{C}$. Depletion of divalent cations by addition of $5 \mathrm{mmol} / \mathrm{l}$ EDTA or EGTA had no significant effect on trophozoite attachment compared with controls in HSCB without chelating agents (EDTA, 115 $(16.7) \%$ and EGTA, $123(25.0) \%$ control; mean $(\mathrm{SE}), \mathrm{n}=10)$.

Trophozoites attached to isolated epithelial cells from rat jejunum, ileum and colon, although parasites attached significantly less avidly to colonic epithelial cells compared with jejunal enterocytes (Fig. 4). Trophozoites attached similarly to enterocyte suspensions consisting predominantly of villus cells to those enriched for crypt cells $(20 \cdot 0(2 \cdot 1) \%$ and $23.3(2 \cdot 2) \%$ attached, respectively; mean (SE), $\mathrm{n}=10$ ).

Giardia trophozoite attachment to rat enterocytes was inhibited in a dose-dependent manner by D-mannose, D-glucose and the polymannose glycopeptide, mannan (Fig. 5), supporting the view that attachment to enterocytes in this in vitro system is 


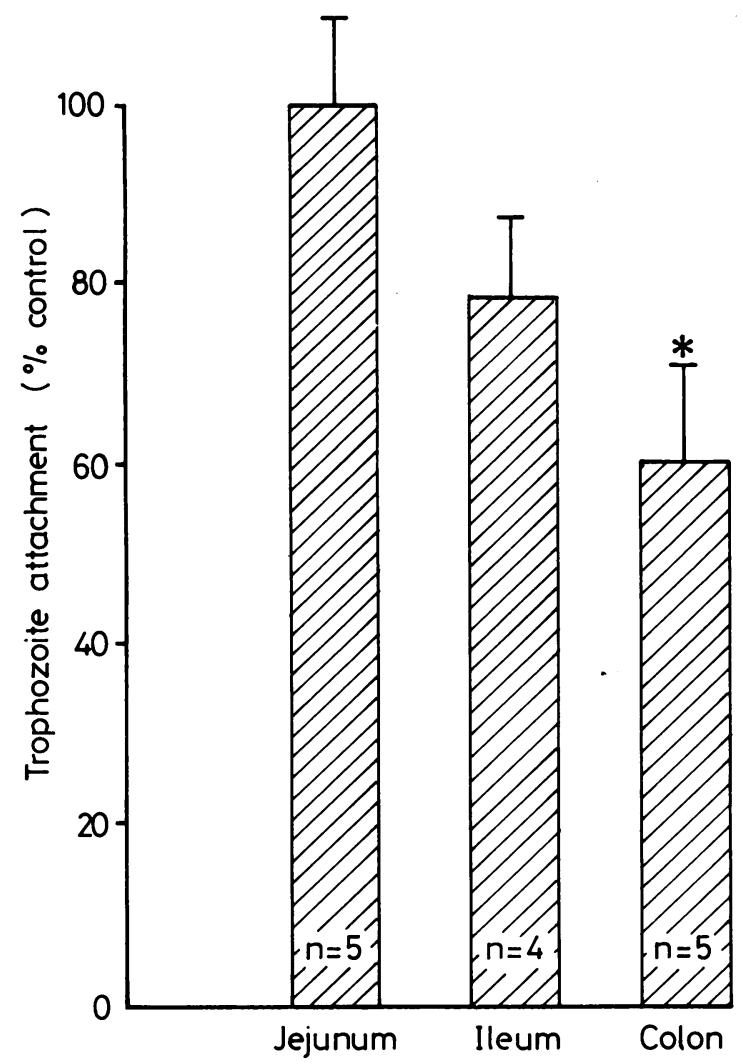

Fig. 4 Attachment of Giardia trophozoites (mean (SE), $n=3$ ) to ileal and colonic rat epithelial cells expressed as a percentage of attachment to jejunal enterocytes (control). ${ }^{*} p<0.01$ compared with attachment to jejunal enterocytes.

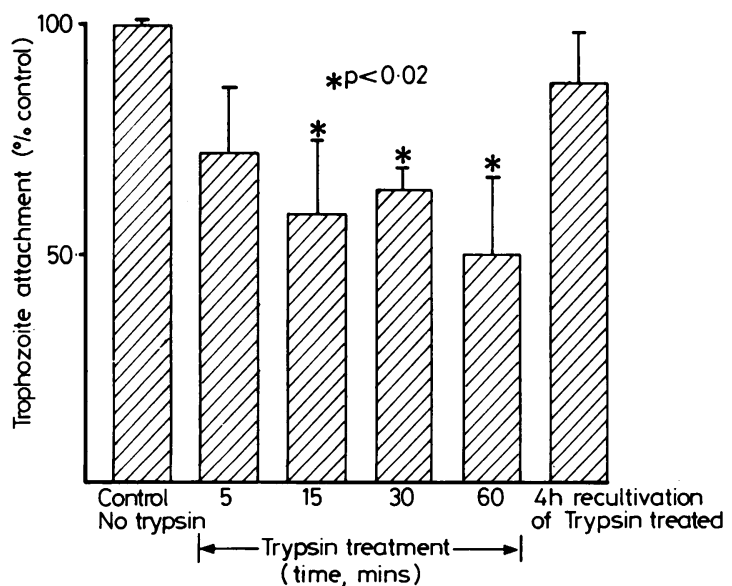

Fig. 6 Effect of trypsin treatment $(1 \mathrm{mg} / \mathrm{ml}$ for 5-60 $\mathrm{min})$ of Giardia trophozoites on attachment to rat jejunal enterocytes. Trypsin treatment reduced the proportion of trophozoites attached, but this was restored to normal by recultivating trypsinized trophozoites in TYI-S culture medium for $4 h .{ }^{*} p<0.02$ compared with control.

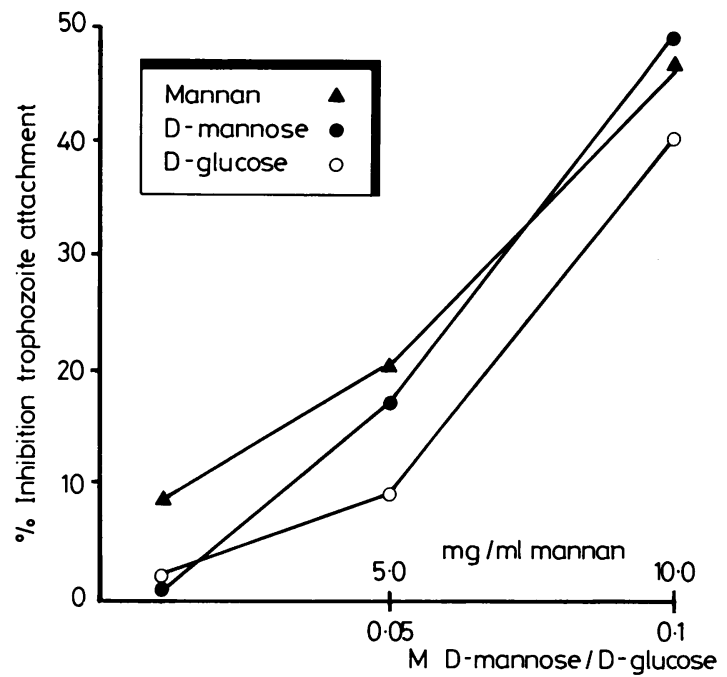

Fig. 5 Concentration dependent inhibition of attachment of Giardia trophozoites to rat jejunal enterocytes by monosaccharides, D-mannose and D-glucose and the polymannose glycoprotein, mannan.

mediated by a parasite surface lectin with specificity for D-mannosyl residues. Attachment was inhibited less effectively by $0 \cdot 1 \mathrm{M}$-methyl D-mannoside $(27 \cdot 8$ $(9.9) \%$ inhibition of trophozoite attachment), and the mannose containing glycopeptide, ovomucoid $(26 \cdot 0(10 \cdot 1) \%$ at $10 \mathrm{mg} / \mathrm{ml})$, but a broad spectrum of other simple and complex carbohydrates (including D-galactose, $\mathrm{N}$-acetyl glactosamine, $\mathrm{N}$-acetyl glucosamine, L-fucose, lactose, sucrose, arabinose, rhamnose, colominic acid, orosomucoid and hog gastric mucin) failed to inhibit attachment at $\leqslant 0 \cdot 1 \mathrm{M}$.

Giardia enterocyte attachment was substantially reduced by pretreating trophozoites with trypsin (Fig. 6). On returning trypsinised parasites to TYI-S culture medium and reculturing for four hours the ability of trophozoites to attach to enterocytes was restored (Fig. 6). Trophozoite generation time in our laboratory is generally seven to eight hours ${ }^{15}$ thus it would appear that new Giardia lectin is recruited on to the cell surface during reculture rather than by production of a progeny of trophozoites with surface lectin activity. Trophozoite attachment was similar with cytochalasin B-treated trophozoites (15.2 $(2 \cdot 4) \%)$ and control trophozoites incubated in the same concentration of DMSO (13.1 (1.6)\%), although the solvent itself did appear to reduce attachment compared to solvent free incubations $(\mathrm{p}<0 \cdot 01)$.

Trophozoite agglutination titres for anti-Giardia rabbit antiserum, and monoclonal antibodies, GL-1 and GL-2 were 128, 2048, and 512, respectively. At subagglutinating concentrations monoclonal anti- 


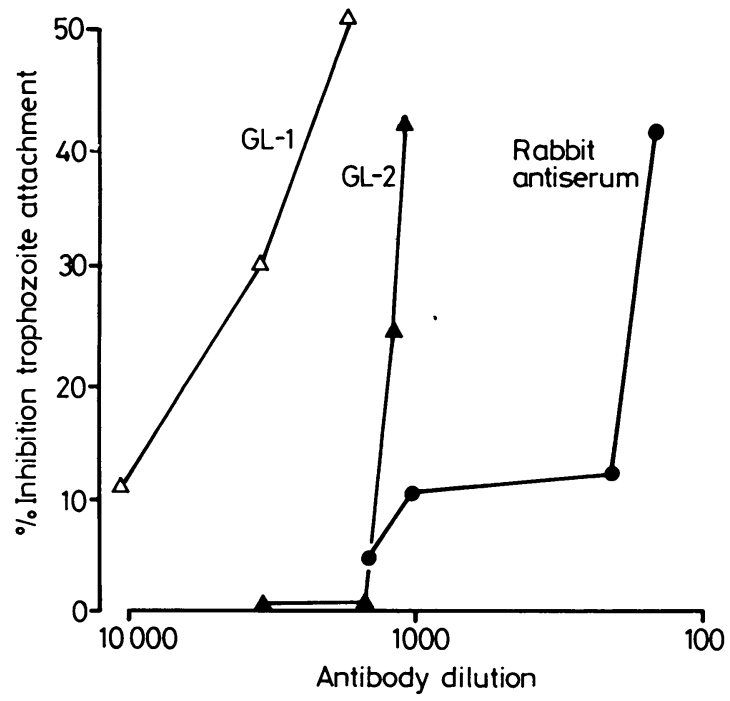

Fig. 7 Concentration-dependent inhibition of attachment of Giardia trophozoites to rat jejunal enterocytes by subagglutinating concentrations of anti-Giardia rabbit serum, and two anti-Giardia mouse monoclonal antibodies, GL-I and $G L-2$.

bodies and the anti-Giardia rabbit antiserum inhibited trophozoite attachment to enterocytes, GL-1 being the most potent (Fig. 7).

\section{Discussion}

These studies show that the human pathogen Giardia lamblia attaches to intestinal epithelial cells with some preference for epithelial cells of the small intestine relative to colon. Attachment was inhibited by monosaccharides and glycoproteins containing D-mannosyl and D-glycosyl residues, suggesting that attachment of the parasite to rat enterocytes is mediated by a surface lectin with specificity for these saccharide residues. These observations are consistent with our previous finding using mammalian erythrocytes as a target system, that Giardia lamblia possesses a D-mannose/D-glucose binding surface lectin. ${ }^{7}$ Relatively high concentrations of monosaccharides were required to inhibit attachment but these were of the same order of magnitude as we reported previously for a mixed agglutination intact cell system. ${ }^{7}$ Attachment was inhibited at $4^{\circ} \mathrm{C}$ which agrees with our previous observation of temperature dependency in the red cell model. ${ }^{7}$ Removal of divalent cations from the incubation medium did not, however, inhibit attachment to enterocytes, an observation which differs from our previous work with erythrocytes where rosette formation with live trophozoites and haemagglutination by non-ionic detergent extracts of Giardia lectin were calcium dependent. There is no immediately obvious explanation for this disparity although it is possible that intracellular calcium stores in the enterocytes were liberated during the incubation and made available to parasites in close apposition to the epithelial cells by virtue of the concentration gradient established by chelating agents.

A recent study has confirmed that Giardia lamblia possesses a lectin with specificity for D-mannosyl residues, haemagglutinating activity being inhibited most effectively by mannose-6-phosphate. ${ }^{19}$ This report suggested, however, that the majority of this lectin was intracellular and required activation by trypsin before it could function as a haemagglutinin. Although the interaction between intact Giardia trophozoites and enterocytes was not investigated in that study, evidence was presented to suggest that trypsin treatment of whole trophozoites could induce lectin activity in sonicates of the parasite. Our data indicate, however, that trypsin removes parasite surface lectin and possibly other surface determinants that are involved in parasite attachment but that these are regenerated during a brief period of axenic culture, suggesting that preformed intracellular lectin is promptly recruited on to the cell surface.

The interaction between trypsin and this surface lectin may be of biological importance as trypsin is plentiful in the proximal small intestine where the parasite spends most of its life. Our data would suggest that trypsin may mediate parasite release from the gut epithelium, but the studies of Lev et al ${ }^{19}$ indicate that trypsin may also function in vivo to activate intracellular pro-lectin. From these in vitro studies it would appear that trypsin would have its most profound effect after death and cell disruption. An alternative explanation for the disparity between the interaction of trypsin and Giardia lectin activity is that the surface lectin described by us $^{7}$ and the predominantly intracellular trypsin-activated lectin described by Lev et al $^{19}$ are in fact different lectins. This would seem unlikely as Lev et al estimated lectin subunit size to be $57000-\mathrm{Mr}$ and we had shown previously a $56000-\mathrm{Mr}$ polypeptide on SDS-PAGE although our affinity purification method did yield a second major band at $78000-\mathrm{Mr} .^{7}$

Our experiments with the microfilament inhibitor cytochalasin B support the view that attachment of the parasite to enterocytes in this system is not dependent on cell motility and contractile proteins of the cytoskeleton, which are known to be present in the ventral disc and other ventral structures. ${ }^{3-5}$ These contractile proteins are also functionally dependent on calcium ions which as stated earlier fail to influence parasite attachment. It has been shown previously, however, that cytochalasin B and 
removal of calcium ions from the incubation medium substantially reduces the ability of Giardia to attach to a polystyrene surface in vitro, ${ }^{16}$ a process which unlike lectin mediated attachment is dependent on contractile properties of the ventral disc and motility of the ventral flagella.

Our finding that anti-Giardia surface antibodies at subagglutinating concentrations can inhibit attachment of Giardia to enterocytes clearly supports the view that surface membrane determinants are involved in this attachment process. At these concentrations heat - inactivated antibody preparations were not cytotoxic as judged by trypan blue exclusion and assessment of parasite motility, nor did they impair growth when added to axenic cultures of Giardia trophozoites (unpublished observations). It is as yet unclear as to whether either of these antibodies is directed towards an epitope on the lectin itself or whether they are merely inhibiting attachment by binding to a lectin associated surface protein.

We consider that these studies provide evidence that a receptor exists on rat intestinal epithelial cells for Giardia mannose binding lectin which may play a part in mediating parasite adherence to host epithelium. Mannose is clearly present in brush border and basolateral membrane glycoproteins, generally considered to occur predominantly in $\mathrm{N}$-glycosidically linked core glycoproteins. ${ }^{20} \mathrm{We}$ have recently shown specific binding of the mannose binding lectin, concanavalin A (conA) to rat enterocytes, when we found approximately $10^{7}$ high affinity receptors per cell $\left(\mathrm{Ka} 3.5 \times 10^{5} \mathrm{M}^{-1}\right)$. $^{21}$ Giardia lectin has some features in common with con $\mathrm{A}$ and may bind therefore to these or similar binding sites.

The authors are indebted to the Wellcome Trust for financial support. MJGF is a Wellcome Trust Senior Lecturer. We also thank Alan Phillips for providing the photomicrographs shown in Figs 1 and 2, and Ms Nikki Thomas and Ms Nicola Herrera for typing the manuscript.

\section{References}

1 Farthing MJG. Receptors and recognition mechanisms in intestinal infection. Trans $R$ Soc Trop Med Hyg 1985; 79: $569-76$.

2 Holberton DV. Attachment of Giardia - A hydrodynamic model based on flagellar activity. $J$ Exp Biol 1974; 60: 207-21.

3 Crossley R, Holberton DV. Characterization of proteins from the cytoskeleton of Giardia lamblia. J Cell Sci 1983; 59: 81-103.

4 Crossley R, Marshall J, Clark JT, Holberton DV. Immunocytochemical differentiation of microtubules in the cytoskeleton of Giardia lamblia using monoclonal antibodies to $\alpha$-tubulin and polyclonal antibodies to associated low molecular weight proteins. I Cell Sci 1986; 80: 233-52.

5 Freely DE, Schollmeyer JV, Erlandsen SL. Giardia spp.: Distribution of contractile proteins in the attachment organelle. Exp Parasitol 1982; 53: 145-54.

6 Mueller JC, Jones AL, Brandborg LL. Scanning electron microscope observations in human giardiasis. In: Scanning electron microscopy 1973. Part III. Chicago: IIT Research Institute, 1973.

7 Farthing MJG, Pereira MEA, Keusch GT. Description and characterization of a surface lectin from Giardia lamblia. Infect Immun 1986; 51: 661-7.

8 Farthing MJG, Keusch GT, Carey MC. Effects of bile and bile salts on growth and membrane lipid uptake by Giardia lamblia: Possible implications for pathogenesis of intestinal disease. $J$ Clin Invest 1985; 76: 1727-32.

9 Weiser, MM. Intestinal epithelial cell surface membrane glycoprotein synthesis. I. An indicator of cellular differentiation. J Biol Chem 1973; 248: 2536-41.

10 Coyler J, Farthing MJG, Kumar PJ, Clark ML, Ohannesian AD, Waldron NM. Reappraisal of the lectin hypothesis in the aetiopathogenesis of coeliac disease. Clin Sci 1986; 71: 105-10.

11 Weinstein R, Silverblatt FJ. Antibacterial mechanisms of antibódy to mannose-sensitive pili of Escherichia coli. J Infect Dis 1983; 147: 882-9.

12 Daifuku R, Stamm WE. Bacterial adherence to bladder uroepithelial cells in catheter-associated urinary tract infection. N Engl J Med 1986; 314: 1208-13.

13 Ravdin JI, Guerrant RL. Role of adherence in cytopathogenic mechanisms of Entamoeba histolytica. J Clin Invest 1981; 68: 1305-13.

14 Phillips AD, France NE, Walker-Smith JA. The structure of the enterocyte in relation to its position on the villus in childhood: an electron microscopical study. Histopathology 1979; 3: 117-30.

15 Farthing MJG, Varon SR, Keusch GT. Mammalian bile promotes growth of Giardia lamblia in axenic culture. Trans $R$ Soc Trop Med Hyg 1983; 77: 467-9.

16 Feely DE, Erlandsen SL. Effect of cytochalasin-B, low $\mathrm{Ca}^{++}$concentration, iodoacetic acid and quinacrine$\mathrm{HCl}$ on the attachment of Giardia trophozoites in vitro. J Parasitol 1982; 68: 869-73.

17 Kohler G, Milstein C. Continuous culture of fused cells secreting antibody of predefined specificity. Nature 1975; 256: 495-6.

18 Edson CM, Farthing MJG, Thorley-Lawson DA, Keusch GT. An 80,000-Mr Giardia lamblia surface protein which is immunogenic in humans. Infect Immun 1986; 54: 621-5.

19 Lev B, Ward H, Keusch GT, Pereira MEA. Lectin activation in Giardia lamblia by host protease: A novel host-parasite interaction. Science 1986; 232: 71-3.

20) Kim YS, Morita A, Miura S, Siddiqui B. Structure of glycoconjugates of intestinal mucosal membranes: role in bacterial adherence. In: Boedeker EC, ed. Attachment of organisms to the gut mucosa. Vol II. Boca Raton: CRC Press Inc, 1984: 99-109.

21 Coyler J, Kumar PJ, Waldron ML, Clark ML, Farthing MJG. Gliadin binding to rat and human enterocytes. Clin Sci 1987; 72: 593-8. 